\title{
Simultaneous PIV and DIC Measurements in a Towing Tank Environment with a Flexible Hydrofoil
}

\author{
G. Jacobi ${ }^{1 *}$, A. Nila ${ }^{2}$ \\ ${ }^{1}$ Delft University of Technology, Ship Hydromechanics and Structures Laboratory, Delft, The Netherlands \\ ${ }^{2}$ LaVisionUK Ltd, United Kingdom \\ *G.Jacobi@tudelft.nl
}

\begin{abstract}
Due to their good mechanical properties composite materials are increasingly applied for the construction of lifting surfaces in the maritime industry. However, besides improving the strength to weight ratio of a structure, the anisotropic material properties can also exhibit bend-twist coupling, when exposed to higher loads. In order to experimentally measure the fluid structure interaction, the object of investigation needs to exposed to the same fluid loadings, as it would experience during operation. To investigate the possibility to obtain simultaneous deformation and flow field measurements in a large hydrodynamic testing facility simultaneous PIV and DIC measurements are performed to obtain the deformation of a flexible NACA 0008 hydrofoil and to measure the flow field in the wing tip region. For the assessment of the performance of the methods two scenarios are presented including tests in stationary conditions with constant angles of attack and forced plunging oscillations. The calibration of both measurement systems is done independently and the wing tip, visible in the PIV images, is used for triangulation to find the position of the wing within the PIV coordinate system. The combination of both measurement techniques allows for an accurate determination of tip vortex center positions with respect to the deformed wing and their evolution downstream of the wing. During forced plunging motions, the phase lag of the wing tip and the influence on the wing tip vortex is observed.
\end{abstract}

\section{Introduction}

Within the last two decades, the maritime industry has been increasingly investigating the use of composite materials and lightweight structures. With composite materials offering higher strength to weight ratios than traditional materials, propellers and tidal turbines were designed with improved vibration properties and higher fatigue life. Especially in performance sailing, but also in the area of passenger transport, composite hydrofoils and lightweight structures lead to the development of fully foiling boats, where the hull is lifted out of the water to reduce its frictional drag. Next to an improved strength to weight ratio, due to their anisotropic properties, composite structures may exhibit bend-twist coupling, which leads to a change of the shape of the structure, when exposed to higher loads. This behavior enables a structure to passively adapt its shape to the incoming flow, therefore allowing for an enhanced hydrodynamic performance to be accounted for in the design process. Under these circumstances the composite material cannot be modeled as a rigid structure since the hydrodynamic and structural analysis are strongly coupled. To consider the fluid-structure-interactions (FSI) in the design phase, but also to develop a further understanding of the hydrodynamics and structural effects due to the interaction, numerical modeling supported by experimental investigations are needed. Despite being interested in the hydrodynamic performance, the fluid structure interaction of hydrofoils at larger scales has only been tested in wind-tunnel facilities, up until now. Marimon Giovannetti (2017) performed simultaneous particle image velocimetry (PIV) and digital image correlation (DIC) measurements in a wind tunnel to investigate the structural deformation of a hydrofoil and its influence on the tip vortex at different angles of attack. While testing at an equivalent Reynolds number, the flow around the hydrofoil can be correctly analyzed in these facilities. However, the fluid dynamic loads are five times smaller compared to testing in water. To expose the foil to the same fluid loadings as it would experience during operation, Marimon Giovannetti (2017) proposed to put further effort in the development 
of a system to simultaneously measure the structural deformation and flow features in a towing tank environment. Several tests to measure structural deformations in small cavitation tunnels have been performed (Young (2018)). However, due to the small dimensions of these, the size of the investigated object is limited. With PIV measurements having become a standard technique for analyzing the flow field during towing tank tests (Falchi et al. (2013)), (Hallmann et al. (2009)), this paper presents a first application of the combination of the two optical measurement techniques, PIV and DIC, in a towing tank environment for the analysis of a flexible hydrofoil.

\section{Test Case}

To test the feasibility of the simultaneous measurement of structural deformations and the herewith associated flow field with an underwater PIV and DIC system, a flexible NACA 0008 hydrofoil is tested in the TU Delft towing tank. For a simplified prediction of the expected deformations, the wing profile is manufactured from silicone, with a $1 \mathrm{~mm}$ aluminum plate integrated at the camber line. To prevent any sideward forces, the foil is tested in a T-foil configuration. The distance of the hydrofoil to the free surface is 5 chord length. The submerged T-foil with a total wing span of $500 \mathrm{~mm}$ is depicted in Figure 1 (left), with Figure 1 (right) showing the outline of the modified NACA 0008 profile with the integrated steel plate. The angle of attack of the foil can be adjusted at the connection of the strut with the foil.
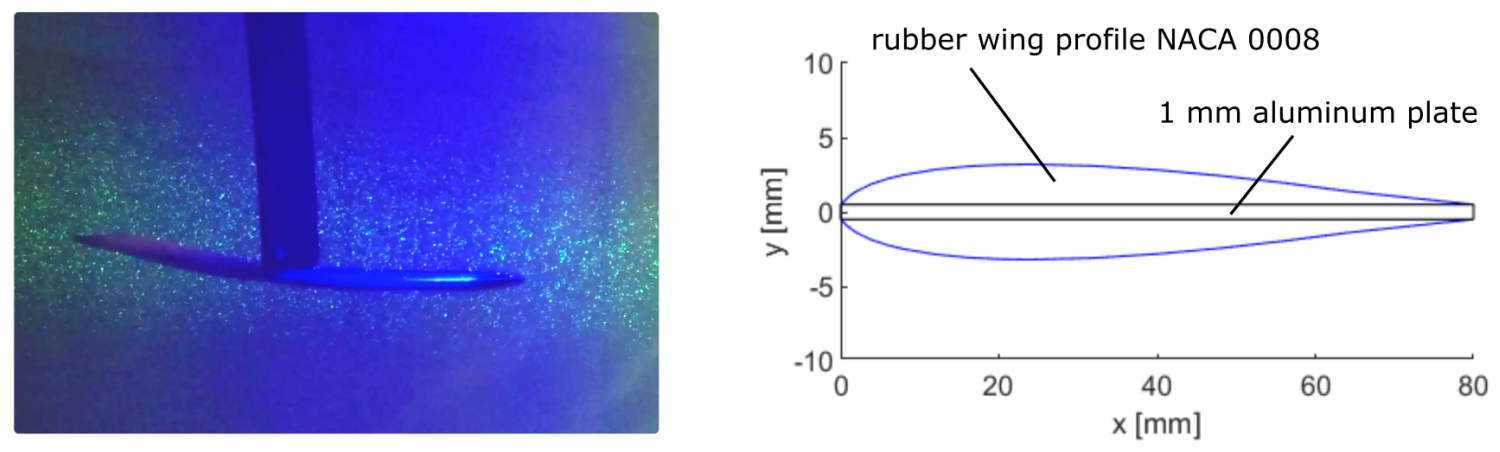

Figure 1: Photograph of the submerged hydrofoil (left) and outline of the modified NACA 0008 profile with a $1 \mathrm{~mm}$ aluminum plate at the camber line (right)

For the assessment of the performance of both measurement techniques, two different test scenarios are presented. During the first scenario, the hydrofoil is towed at $1 \mathrm{~m} / \mathrm{s}$ at different angles of attack, varying from 3 to 9 degrees, to obtain the time averaged deformation of the foil, as well as the associated flow field in the wake of the foil. In a second scenario, the hydrofoil is towed with the same velocity of $1 \mathrm{~m} / \mathrm{s}$, but is subjected to forced sinusoidal heave oscillations at a frequency of $1 \mathrm{~Hz}$. Simultaneous DIC and PIV measurements are used to capture the phase-averaged characteristics of the hydrofoil structure, as well as the flow field in its wake.

\section{Experimental Setup}

Figure 2 (left) gives a general overview of the test setup, showing the towing tank carriage of the towing tank of the TU Delft ship hydrodynamics laboratory with the attached foil and the optical measurement equipment. The towing tank is $150 \mathrm{~m}$ long with a cross section of $4.5 \mathrm{~m}$ width and $2.5 \mathrm{~m}$ depth. A more detailed description of the setup can be found in Figure 2 (right). The flexible hydrofoil, as well as the DIC system are attached to a hexapod mounted on the towing carriage. This allows for the performance of forced oscillatory motions and a repositioning of the hydrofoil in between measurement runs. To capture the deformation of the submerged hydrofoil, the DIC system is fitted into a generic ship hull. The PIV system, including cameras and laser optics is fitted into a torpedo shaped probe which is towed next to the hydrofoil. 


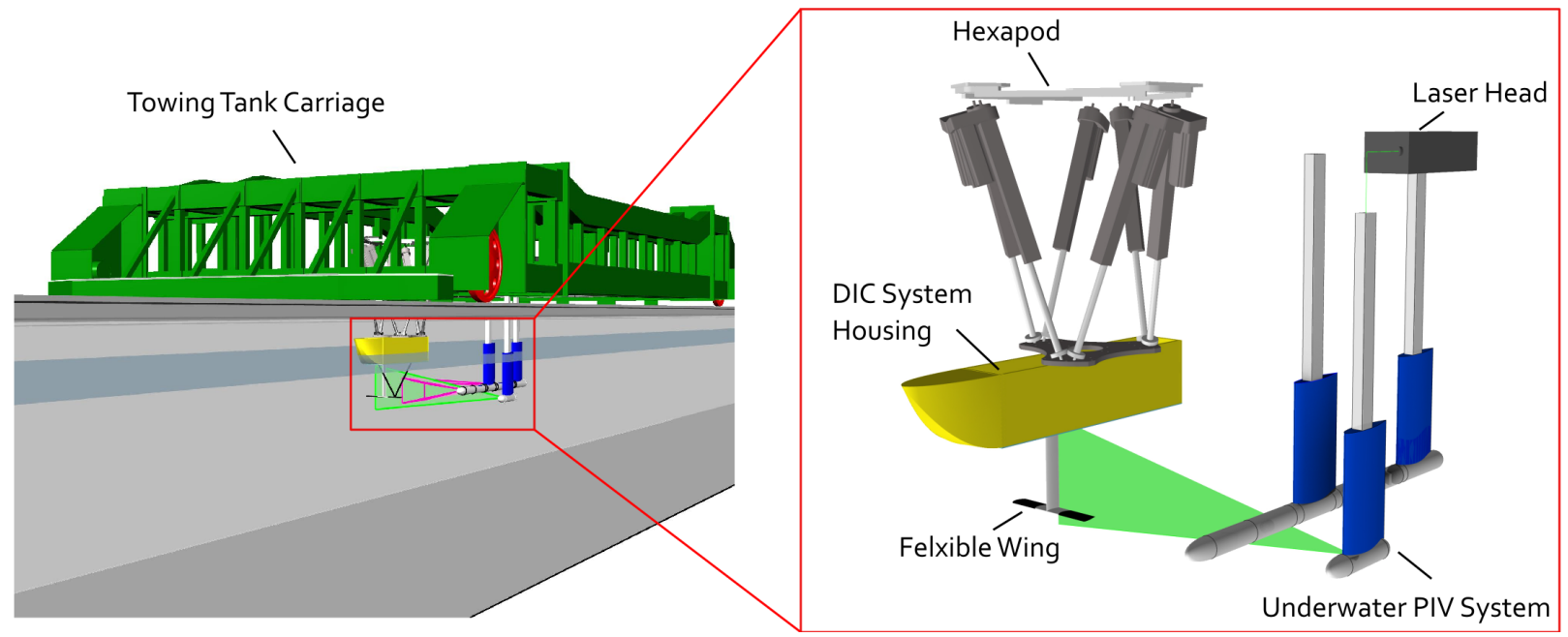

Figure 2: Schematic drawing of the towing tank carriage with the attached measurement configuration (left) and schematic drawing of the detailed measurement setup, including the flexible wing and both optical measurement systems (right)

\subsection{Deformation Measurements}

A schematic overview of the optical DIC setup is presented in Figure 3 (left). Image acquisition is done with two LaVision Imager M-lite 2M cameras both looking at the object of investigation under an angle of 45 degrees through water-filled prisms attached to the acrylic glass bottom. As the DIC system is oscillating together with the hydrofoil, it is guaranteed, that the speckle pattern on the top of the hydrofoil will stay in focus, also with larger oscillation amplitudes. Illumination for the DIC measurements is done with two linear illumination units from LaVision at a wavelength of $450 \mathrm{~nm}$. Recording of the images is done at $25 \mathrm{~Hz}$ for both, the stationary and oscillation tests. To reduce the interference of the measurement systems, the DIC cameras are equipped with bandpass filters. Calibration is done with a two-level double-sided $3 \mathrm{~d}$ calibration plate of $200 \times 200 \mathrm{~mm}$. During the calibration of the DIC system, the target is horizontally aligned with the wing. Prior to mounting the optical setup to the hexapod, the latter has been carefully aligned with the towing tank coordinate system. For measurement of the hydrofoil deformations, a random speckle pattern is a applied to its surface. The pattern is applied manually with spay paint. The final image speckle size is approximately 2 pixels with a standard deviation of \pm 1.5 pixels. Figure 3 (right) shows a recorded image of the hydrofoil with the speckle pattern. Data acquisition and the calculation of deformations from the stereo-image pairs is conducted with the Strain Master package from LaVision. After an initial triangulation to find the initial surface shape, the deformation is calculated with a subset size of 27 pixels and a step size
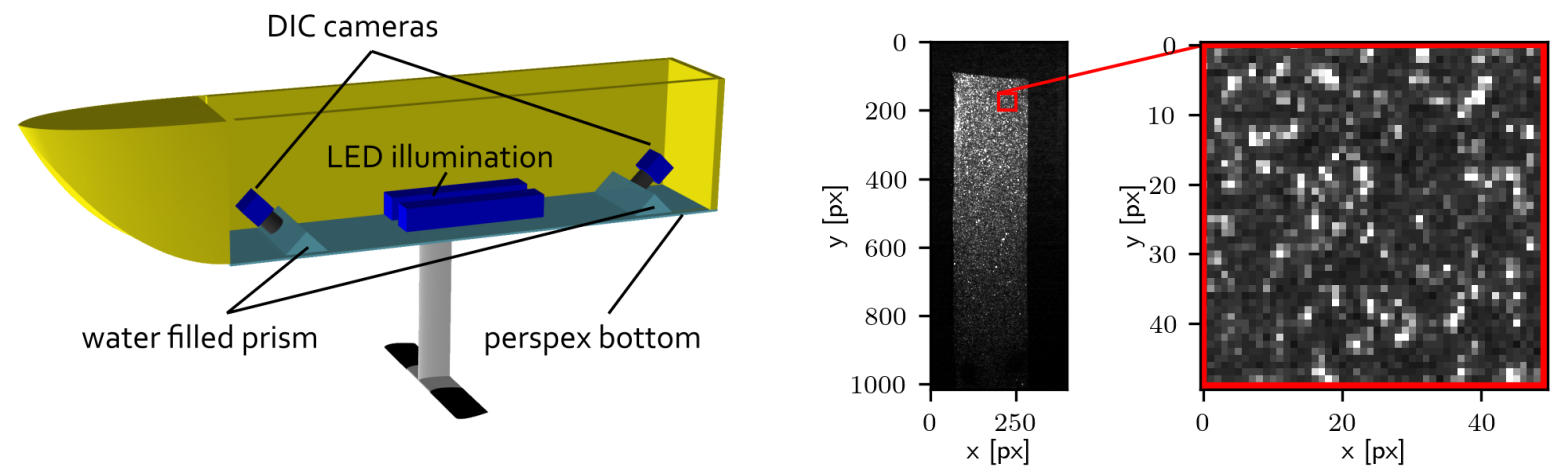

Figure 3: Detailed schematic overview of the DIC setup (left) and recorded raw image of the flexible wing with the applied speckle pattern (right) 
of 6 pixels. At a resolution of 5 pixels $/ \mathrm{mm}$ this results in a spatial resolution of approximately $1.25 \%$ of the chord length. To estimate the uncertainty of the obtained deformations, a series of images is recorded without moving the wing. A comparison of the obtained deformations results in a maximum uncertainty of $0.125 \%$ of the chord length.

\subsection{Flow Field Measurements}

The stereo-PIV system for the measurement of the flow field in the wake region of the hydrofoil is fitted into a torpedo shaped underwater housing, which together with the strut for the laser optics is towed next to the hydrofoil (Figure 2 (right)). A detailed description of the torpedo and its modules is depicted in Figure 4 (left). Image acquisition is done with two LaVision Imager MX cameras with a resolution of 4 megapixels equipped with lenses of $28 \mathrm{~mm}$ focal length which are mounted to a motorized Scheimpflug adapter with attached remote focus and aperture control. In front of the cameras water filled mirror sections are integrated into the torpedo, making both cameras facing the measurement area under an angle of 30 degrees. Recording of the double frame images is done at $25 \mathrm{~Hz}$ and synchronized with the DIC system via the LaVision Programmable Timing Unit (PTU). As the measurement plane is oriented perpendicular to the towing direction, the time between two successive images is limited by the out-of-plane particle motion, resulting in a time separation of $\Delta \mathrm{t}=900 \mu \mathrm{s}$ at a towing tank carriage speed of $1 \mathrm{~m} / \mathrm{s}$.
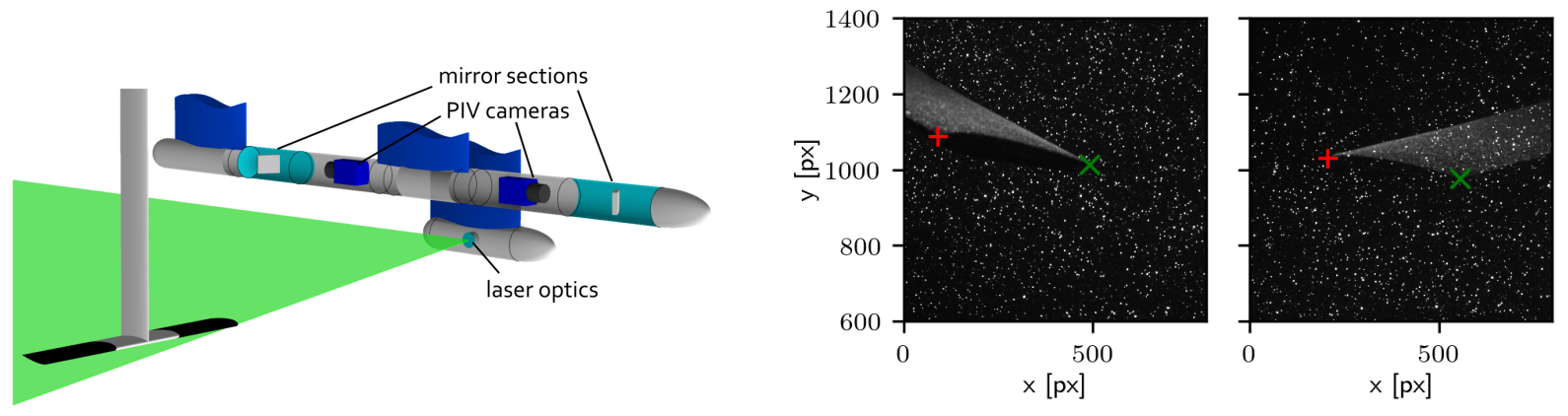

Figure 4: Detailed schematic overview of the PIV setup (left) and recorded raw PIV images with the detected reference points which are used for triangulation

Illumination of the measurement area is done with a $50 \mathrm{~mJ}$ Litron Nano-PIV laser, operating at a wavelength of $532 \mathrm{~nm}$. The laser beam is guided into a separate strut that includes the beam focusing optics and the cylindrical lens for generation of the sheet. The PIV system has a stand-off distance of $1000 \mathrm{~mm}$ to the center of the measurement area, resulting in a resolution of approximately 6 pixels $/ \mathrm{mm}$. To guarantee a uniform distribution of the $50 \mu \mathrm{m}$ polymer (Vestosint) particles in the measurement area, a retractable seeding rake is mounted in front of the carriage. Prior to releasing the particles into the towing tank in between measurement runs, they are premixed in a high shear flow to prevent clustering. After every measurement run the tank is reseeded and a waiting time of 25 minutes allows the water to settle in between runs. Calibration is done with a two-level double-sided $3 \mathrm{~d}$ calibration plate of $320 \times 320 \mathrm{~mm}$. The PIV calibration target is carefully aligned in an iterative process with the moving direction of the carriage and the laser sheet. Mounting the calibration plate to the hexapod allows for an accurate repositioning of the plate during the calibration process. A relation between the DIC and PIV coordinate systems is established after calibration with fiducial points on the tip of the flexible wing and the usage of the pinhole calibration model. This allows for a triangulation of the identified points, to find their position on the PIV coordinate system. Figure 4 (right) shows an example of raw images recorded with both cameras and the identified reference points on the leading and trailing edge on the wing tip which are used for triangulation. As no additional filters were used for the PIV cameras, the illuminated wing is visible in te background of the images. However, due to the limited focal range of the PIV cameras, it is not in focus and can be removed using a sliding average filter which is subtracted from the image prior to vector calculation. The velocity vectors are calculated in an iterative procedure, starting with an interrogation window size of $64 \times 64$ pixels with $50 \%$ overlap with a final interrogation window size of $24 \times 24$ pixels with $75 \%$ overlap. This results in a spatial resolution of 1 vector/mm which is $1.25 \%$ of the hydrofoil chord length. Figure 5 shows the final result of the matched coordinate systems with the reconstructed wing surface from the DIC measurements and vorticity field in 
the tip vortex region obtained in multiple planes behind the wing for an angle of attack of 3 degrees. The PIV results are obtained from averaging over 400 vector images. The obtained results clearly show the change of the hydrofoil shape due to the hydrodynamic loads and the diffusion of the tip vortex downstream of the hydrofoil.

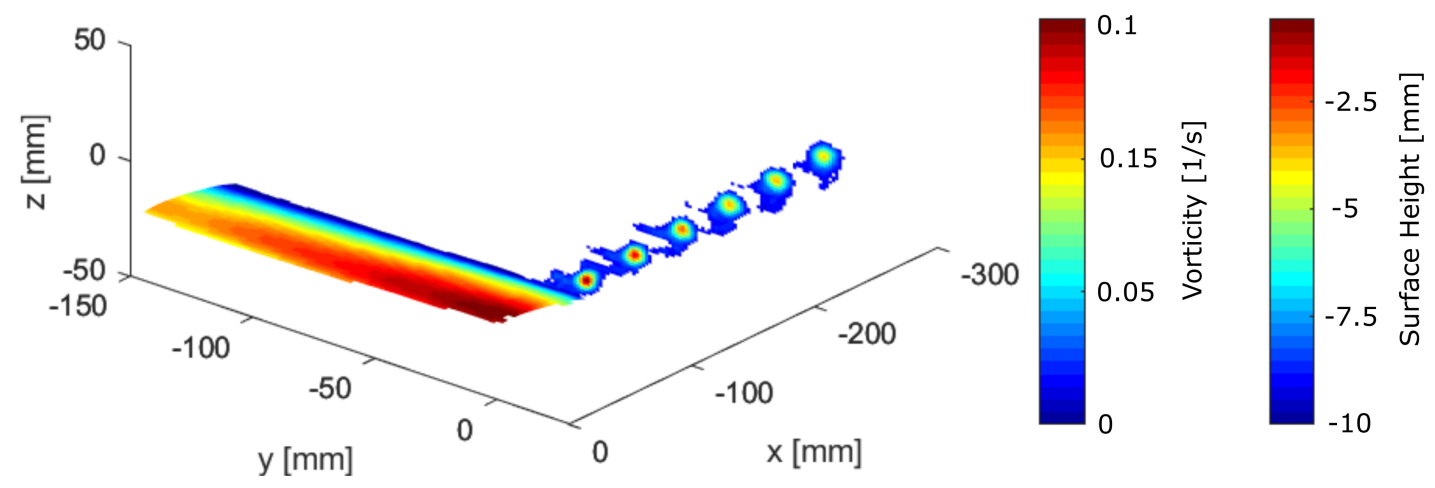

Figure 5: Reconstructed upper wing surface from DIC measurements with time-averaged vorticity fields obtained from PIV measurements at multiple stations behind the wing

\section{Results}

\subsection{Steady Motion}

For a detailed analysis of the influence of the wing shape on the tip vortex the exact shape and deformation of the wing and its position relative to the tip vortex has to be known. For this purpose, the wing shape is reconstructed for angles of attack of 3, 6 and 9 degrees, obtained at a constant translation of $1 \mathrm{~m} / \mathrm{s}$. Figure 6 (left) shows the reconstructed wing shapes for the tested angles of attack, clearly indicating the upward displacement of the wing tip and the twist which are increasing with increasing angle of attack due to the hydrodynamic load. By considering the wing as an idealized beam with one fixed and one free end and the lift force as a distributed load, a least-squares fit of the bending curve allowed an estimation of the lift forces. The results are show in Figure 6 (right), which shows the expected linear distribution within the considered range of angles of attack. A detailed overview of the leading edge displacement which was considered for
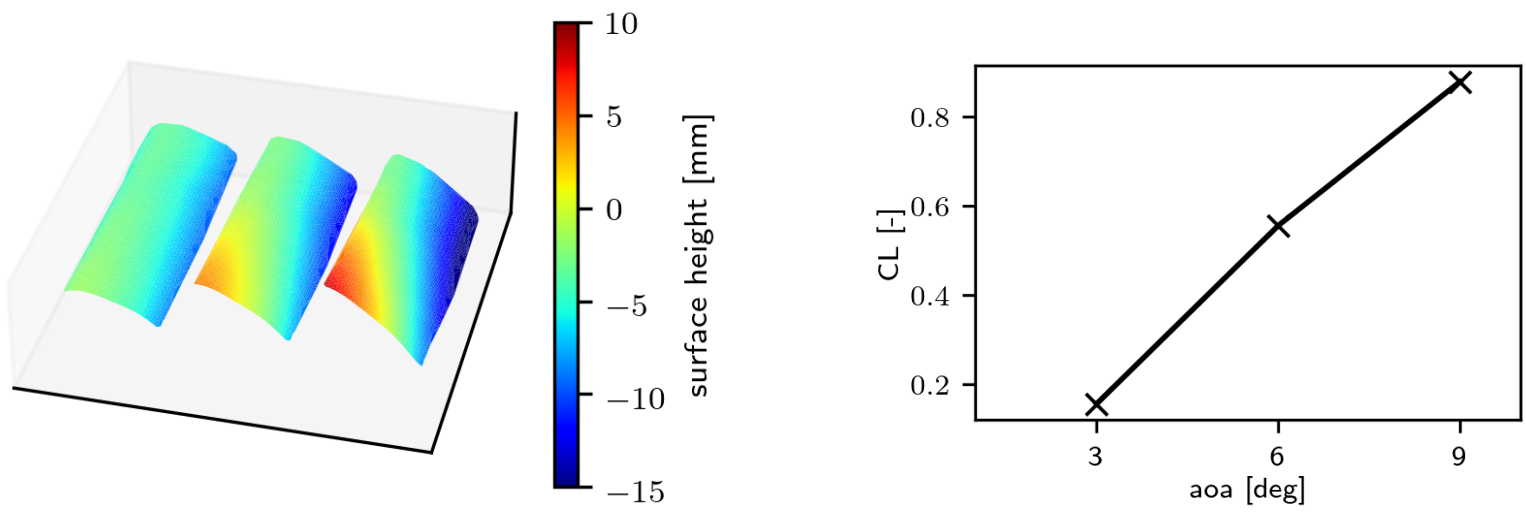

Figure 6: Reconstructed upper wing surfaces at 3,6 and 9 degrees of angle of attack (left) and approximated lift coefficient obtained from the deformation measurements 

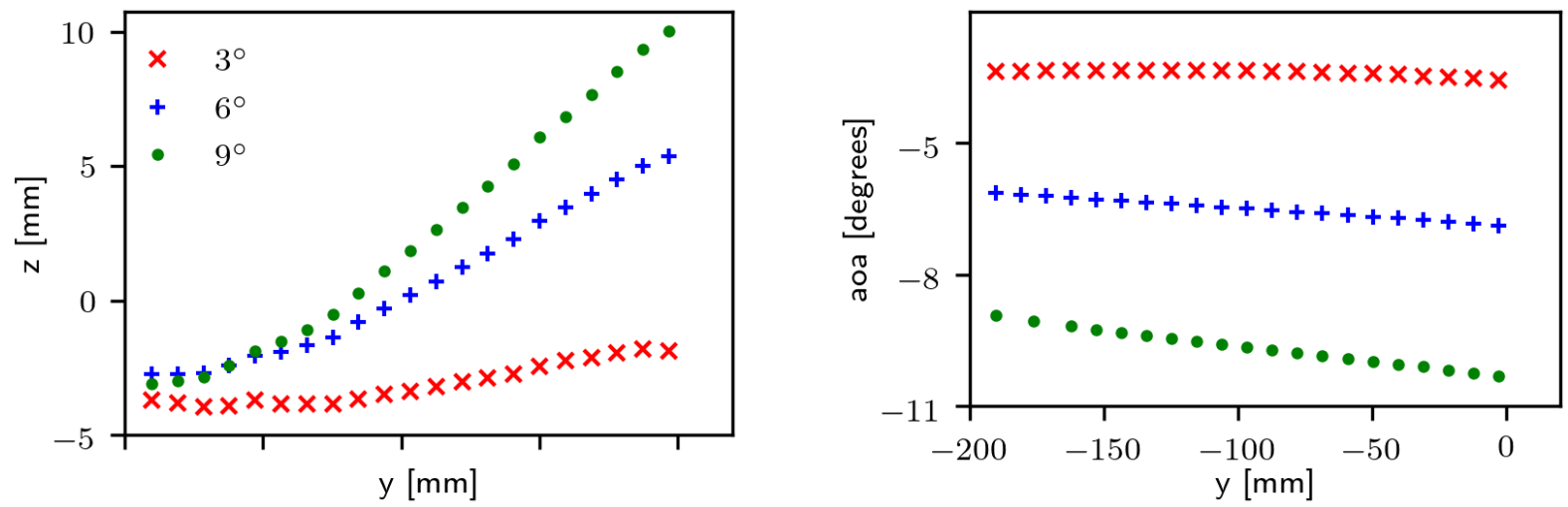

Figure 7: Spanwise distribution of the vertical position of the leading edge (left) and angles of attack (right) for angles of attack of 3,6 and 9 degrees

the estimation of forces is presented in Figure 7/(left). Figure 7)(right) shows the twist angle along the span of the wing which is calculated from the difference of measured leading and trailing edge positions. While at an angle of attack of 3 degrees, the amount of twist is negligible, at 9 degrees, the wing tip is twisted by approximately 2 degrees.

Having obtained the position of the wing within the PIV coordinate system, the flow field of the tip vortex can be accurately related to the deformed wing tip position. Figure 8 shows the vertical component of the flow field in the wing tip region two chord lengths behind the wing for angles of attack of 3,6 and 9 degrees. The continuous and the dashed line indicate the leading and trailing edge positions. To determine the position of the tip vortex center with respect to the deformed wing tip, the vortex center is estimated by finding the maximum of the $\Gamma_{1}$ distribution which is suggested by Graftieaux et al. (2001):

$$
\Gamma_{1}(P)=\frac{1}{N} \sum_{S} \frac{\left(P M \wedge U_{M}\right) \cdot z}{\|P M\| \cdot\|U M\|}
$$

with $N$ being the number of points $M$ within the rectangular area $S$ with center $P$ and $U_{M}$ describing the velocity vector. The locations of the identified vortex centers from the measured flow field are included into Figure 8. Having obtained the flow field in a total of 14 positions behind the wing within the range of $x / c=1-14$, the position of the vortex center at these positions along the wake is obtained. Results are
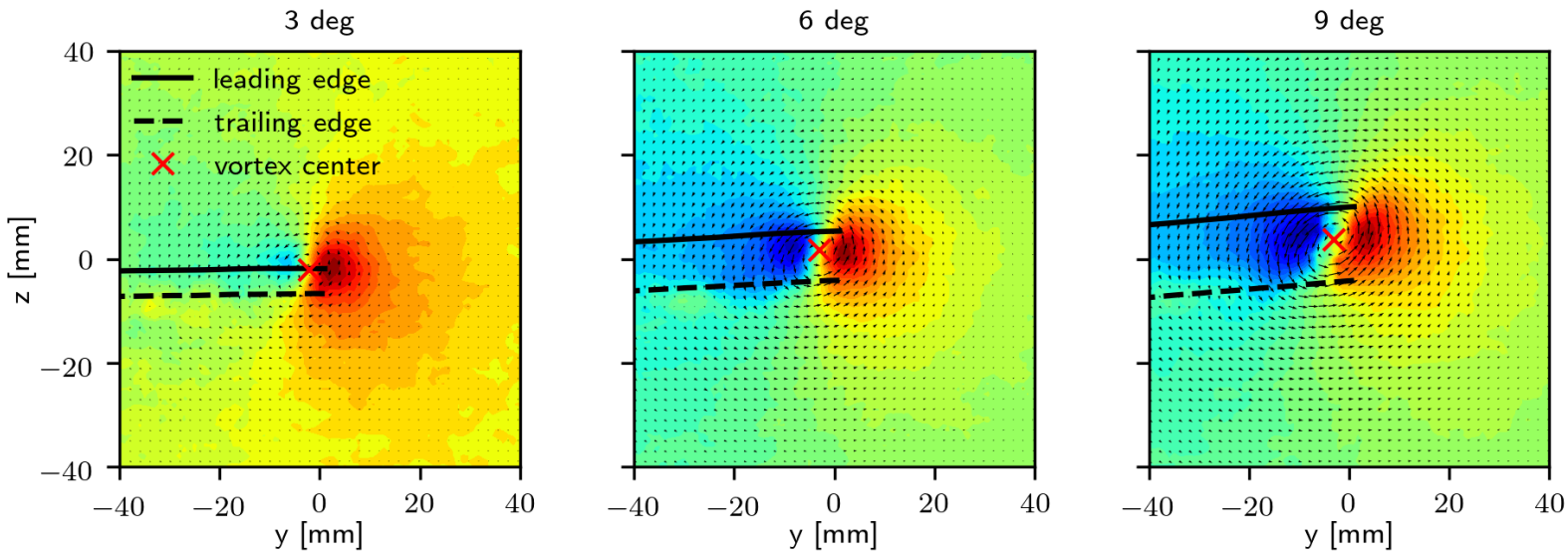

Figure 8: Measured flow field in the wing tip region at $\mathrm{x} / \mathrm{c}=2$ for angles of attack of 3, 6 and 9 degrees with leading and trailing edge positions and the detected center of the tip vortex 
presented in Figure 9 (left), showing the evolution of spanwise and vertical positions of the vortex centers along the wake. Due to its robustness, the vortex center identification performs well over the whole length of the wake from $x / c=1$ to $x / c=14$, clearly reconstructing the inward motion of the tip vortices towards the root of the wing, which is increasing with increasing angle of attack. The vertical position of the tip vortices is presented in Figure 9 (right) together with the wing profiles and their vertical displacement at the wing tip which is obtained from the DIC measurements. The vertical tip vortex displacement increases with the upward displacement of the wing tips. While at 3 degrees, the downward motion of the vortices along the wake is negligible, it becomes clearly visible with higher angles of attack.
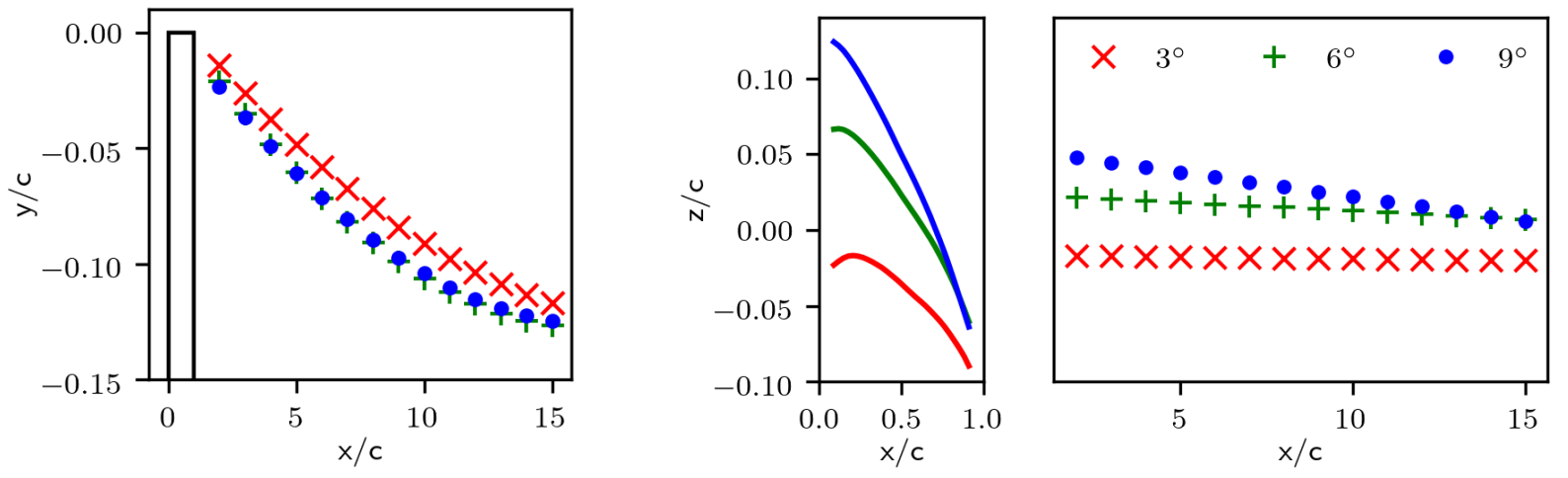

Figure 9: Evolution of the detected tip vortex centers downstream of the flexible wing

\subsection{Forced Oscillations}

The combination of the measurement of structural deformations together with the associated flow field becomes of even more importance when the position of the structure changes over time. The instantaneous position of the structure with respect to the flow field has to be known for an analysis of their interaction. Phase-averaged oscillation tests are performed to investigate the temporal evolution of the wing tip vortex and its position with respect to the wing which is undergoing a forced plunging motion. To capture a sufficient amount of time steps over one oscillation cycle, the oscillation frequency was set to $1 \mathrm{~Hz}$. The angle of attack of the wing was set to 0 degrees. At an oscillation amplitude of $0.024 \mathrm{~m}$ this leads to a maximum apparent angle of attack of 8.5 degrees at 90 and 270 degrees of the oscillation cycle. The results from the forced oscillation tests are depicted in Figure 10. Figure 10 (top) shows the temporal evolution of the vertical position of the hydrofoil at the root and the wing tip. Furthermore, the displacement of the wing tip with reference to its non-deformed position is given. As discussed by Heathcote et al. (2008), the spanwise flexibility and the deformation resulting from the forced plunging motion results in an equivalent motion of the wing tip, which is varying in phase. Due to the low stiffness of the wing used during the experiment, the vertical bending of the wing tip is shifted by 90 degrees and is proportional to the temporal evolution of the apparent angle of attack. The maximum deformation observed at the wing tip is comparable to the deformation observed during the static tests. The deformation results in a phase-lag of the vertical position of the wing tip with respect to the hexapod motion. The influence of the plunging motion on the flow field is depicted in Figure 10 (bottom), showing the vorticity contours at two chord lengths behind the hydrofoil at selected phase-angles. The phase-averaged vorticity fields are obtained from a total of 20 oscillation cycles. Taking into account the acceleration of the towing tank carriage and the limited length of the tank this is the maximum number of oscillations which could be performed within one measurement run. As the hydrofoil is towed at zero angle of attack, the rotation direction of the tip vortex changes due to the downward ad upward motion. Due to the phase-lag of the wing tip with respect to the hexapod motion, the development of the tip vortex is also delayed. 

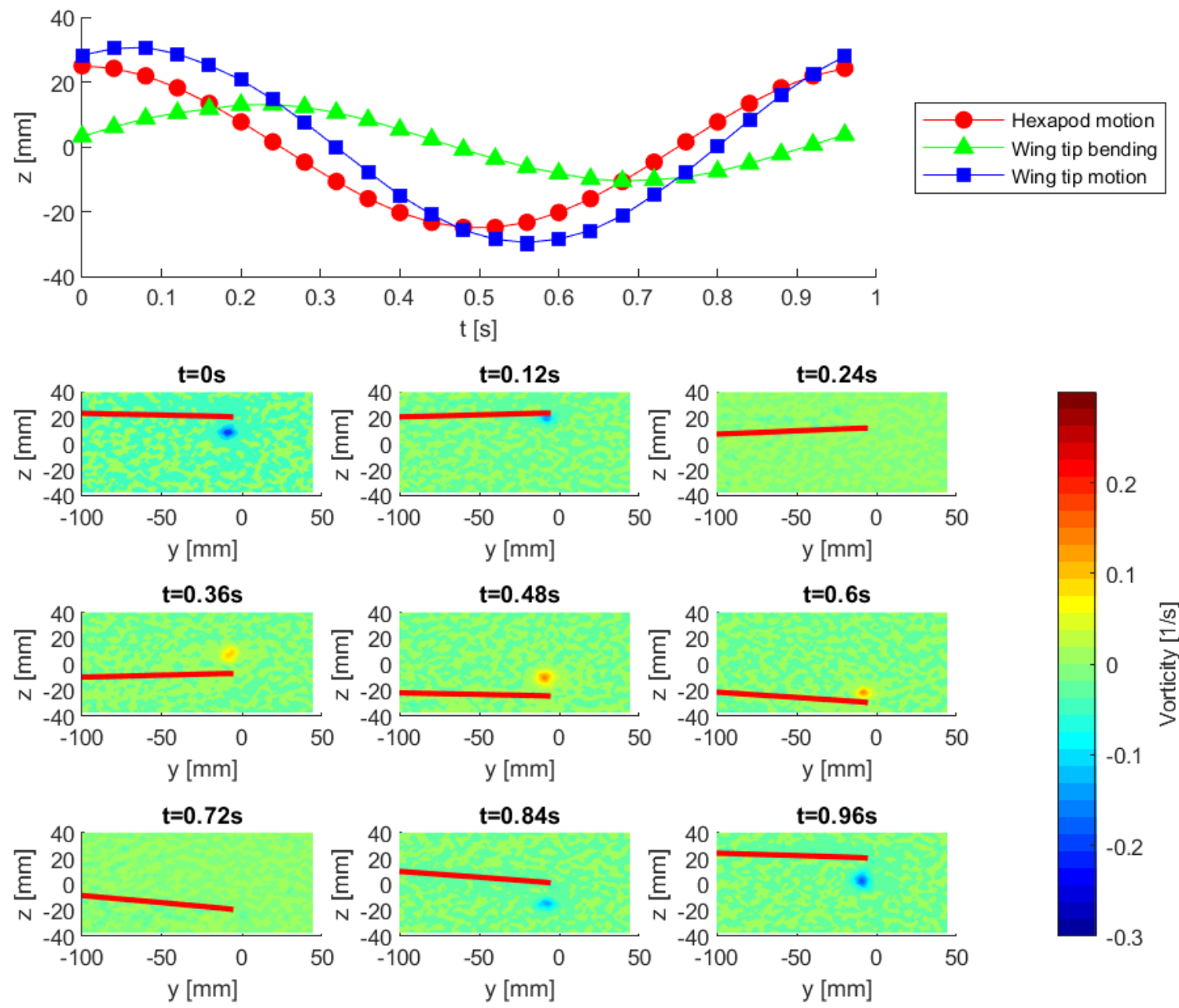

Figure 10: Temporal evolution of the hexapod motion, wing tip bending and total wing tip motion (top) and wing deformation with associated flow field two chord lengths behind the hydrofoil at selected phase angles (bottom).

\section{Conclusion}

With increasing use of composite materials in the maritime sector, more fluid structure interaction measurements in towing tanks will be needed in the future to simultaneously assess the structural deformation, as well as the measurement of the flow field. While up to now, the fluid structure interaction of hydrofoils has been only assessed in wind tunnel facilities, this paper presents a first application of simultaneous PIV and DIC measurements in a towing tank environment, where the object of investigation is exposed to the same fluid loadings as it would experience during operation in water. To obtain a sufficient number of images for the determination of the time- and phase-averaged characteristics of the structure and the flow field, the optical setup cannot be located outside of the towing tank, measuring the flow field at a single location of the tank, when the carriage passes the stationary measurement setup. Instead, all optical components have to be attached to the towing tank carriage to be towed next to the investigated object. While both measurement techniques are of optical nature, the interference of both has to be reduced, selecting different wavelength for the illumination of the speckle pattern on the structure and the particles in the flow field. When combining different optical measurement techniques a common coordinate system is necessary. Especially, when analyzing flexible structures, an exact knowledge of the position is vital for a low spatial uncertainty. The combination of DIC and PIC with both having high spatial resolutions and the usage of fiducial markers allows for a highly accurate determination of the position of the measured flow field vectors with respect to the deforming wing. 


\section{References}

Falchi M, Grizzi S, Aloisio G, Felli M, and F DF (2013) Critical issues in the application of Stereo-PIV in large hydrodynamic facilities : study of a catamaran in steady drift. 10TH Internationnal symposium on particle image velocimetry -PIV13

Graftieaux L, Michard M, and Nathalie G (2001) Combining PIV, POD and vortex identification algorithms for the study of unsteady turbulent swirling flows. Measurement Science and Technology 12:1422-1429

Hallmann R, Tukker J, and Verhulst M (2009) Challenges for Piv in Towing Facilities. AMT'09, Nantes pages $10-22$

Heathcote S, Wang Z, and Gursul I (2008) Effect of spanwise flexibility on flapping wing propulsion. Journal of Fluids and Structures 24:183-199

Marimon Giovannetti L (2017) Fluid structure interaction testing, modelling and development of passive adaptive composite foils. Ph.D. thesis. University of Southampton

Young Y (2018) Load-dependent bend-twist coupling effects on the steady-state hydroelastic response of composite hydrofoils. Compos Struct 189:pp. 398-418 